\title{
Stresses and deformations of an eccentric cylindrical cam during hydro-joining
}

\author{
Kelin Wang ${ }^{1}$, Wei Zhang ${ }^{1}$, Yihua Dou ${ }^{2}$, Xiaozeng Wang ${ }^{3}$, Yinping Cao ${ }^{2, *}$ \\ ${ }^{1}$ Tarim Oilfield Company, PetroChina, Korla, 841000 , China \\ ${ }^{2}$ Xi'an Shiyou University, Xi'an, 710065, China \\ ${ }^{3}$ Jiaying University, Meizhou, 514015, China
}

\begin{abstract}
The stresses and deformations of an eccentric cylindrical cam during the process of hydro-joining have been calculated in orthogonal curvilinear coordinates according to the mechanical model of the cam and governing equations in terms of appropriate complex potentials with suitable boundary conditions. The radial and shearing stress coefficients determined for an example cam are far less than that of the tangential stress during hydro-joining. The tangential stress coefficient of the outer surface of the cam is greater than that of the internal surface when the polar angle exceeds a particular value. The position of the maximum value of the radial stress coefficient is located on the internal surface of the cam, and the maximum shear stress coefficient is located between the inside and outside surfaces of the cam. The cam deformations on the internal and external surfaces under internal pressure respectively attain maximum values at particular angles. The maximum values of the radial and y-directional deformations are located at the position of the minimum wall thickness. The radial deformations determined for an example cam are far larger that the tangential deformations during hydro-joining. The errors between the theoretical and numerical solutions for the tangential stress and the y-directional deformation are both very small.
\end{abstract}

Key Words: Eccentric cylindrical cam, stress, deformation, complex potential, orthogonal curvilinear coordinates.

\section{Introduction}

When the stresses exceed the strength of cam material under the action of the contact pressure, the failure of the cam will occur[1-2]. An eccentric cylindrical cam (ECC) has a cylindrical structure of variable cross-section, and the basic equations governing stresses and deformations are therefore variable coefficient differential equations, which are very difficult to solve[3-4]. To obtain the stresses and deformations of an ECC, many researchers have adopted numerical methods based on the finite element model of the cam[5-8].

In this work, we determine the positions and values of the maximum stress and deformation of an example ECC. We also develop the finite element model of the cam and determine the numerical solutions for the stress and deformation of the cam.

\section{Orthogonal curvilinear coordinates}

Giving definite constant values to $\xi$ and $\eta$, $f_{1}(x, y)=\xi$ and $f_{2}(x, y)=\eta$ will represent two curves which will intersect when $f_{1}(x, y)$ and $f_{2}(x, y)$ are suitable functions. Different values of $\xi$ and $\eta$ will yield different curves and a different point of intersection. Thus, each point in the xy plane will be characterized by definite values of $\xi$ and $\eta$. $\xi$ and $\eta$ may be regarded as coordinates of a point. Because given values of $\xi$ and $\eta$ define the point by means of two intersecting curves, they are called curvilinear coordinates. Polar coordinates are a special case of curvilinear coordinates which have proved very useful for problems of concentric circular boundaries. An ECC has two non-concentric circular boundaries, and such problems typically require curvilinear coordinates, $\xi$ and $\eta, 16$ defined as

$$
z=i a \cdot \operatorname{cth}\left(\frac{\zeta}{2}\right)
$$

Where a is a real constant, $\zeta=\xi+i \eta$, and $z=x+i y$.

\footnotetext{
* Corresponding author: caoyinping02@xsyu.edu.cn
} 


\section{Stresses of an ECC in orthogonal curvilinear coordinates}

\subsection{Stress and stain equations in terms of complex functions}

Substituting $\xi=\xi_{0}$ in Equation (1), we obtain $x^{2}+\left(y-a c t h \xi_{0}\right)^{2}=a^{2} \operatorname{csch} \xi_{0} \quad$.The following equations are for the stresses and deformations of an ECC in terms of complex functions in orthogonal curvilinear coordinates.

$$
\begin{gathered}
\sigma_{\xi}+\sigma_{\eta}=2\left[\psi^{\prime}(z)+\overline{\psi^{\prime}(z)}\right]=4 \operatorname{Re} \psi^{\prime}(z) \\
\sigma_{\eta}-\sigma_{\xi}+2 i \tau_{\xi \eta}=2 e^{2 i \alpha}\left[\bar{z} \psi^{\prime \prime}(z)+x^{\prime \prime}(z)\right] \\
2 G\left(u_{\xi}-i u_{\eta}\right)=e^{i \alpha}\left[(3-) v /(1+v) \bar{\psi}(\bar{z})-\bar{z} \psi^{\prime}(z)-x^{\prime}(z)\right] \text { (4) }
\end{gathered}
$$

\subsection{Calculation formulas for deformation}

Taking the same complex potentials as employed in the calculation of the stress components, we calculate the radial and tangential deformations of an ECC and obtain the expressions for the radial and tangential deformations of an ECC owing to the expansion of the axial tube in the process of hydro-joining.

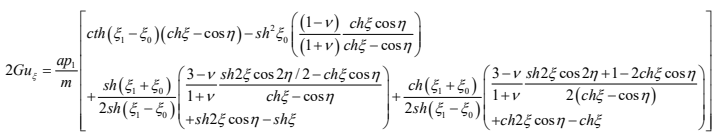

$$
\begin{aligned}
& 2 G u_{\eta}=\frac{a p_{1}}{m}\left[\begin{array}{l}
\frac{c h\left(\xi_{1}+\xi_{0}\right)}{2 \operatorname{sh}\left(\xi_{1}-\xi_{0}\right)}\left(\begin{array}{l}
\frac{v-3}{1+v} \frac{2 \operatorname{ch} \xi \sin \eta-\operatorname{sh} 2 \xi \sin 2 \eta}{2(c h \xi-\cos \eta)} \\
+\operatorname{sh} 2 \xi \sin \eta
\end{array}\right)-\frac{1}{2} \operatorname{sh} h^{2} \xi_{0}\left(\frac{2(1-v)}{1+v} \frac{\operatorname{sh} \xi \sin \eta}{\operatorname{ch} \xi-\cos \eta}\right)+ \\
\frac{\operatorname{sh}\left(\xi_{1}+\xi_{0}\right)}{2 \operatorname{sh}\left(\xi_{1}-\xi_{0}\right)}\left(\frac{v-3}{1+v} \frac{\operatorname{sh} \xi \sin \eta-\operatorname{ch} 2 \xi \sin 2 \eta / 2}{\operatorname{ch} \xi-\cos \eta}+\operatorname{ch} 2 \xi \sin \eta\right)
\end{array}\right]
\end{aligned}
$$

\section{Examples}

\subsection{Stresses of the ECC}

As an example, we set the radius of the outer circle of an $E C C$ at $48 \mathrm{~mm}$, the radius of the inner circle at $30 \mathrm{~mm}$, the distance between their centers at $5.4 \mathrm{~mm}$, and the height of the ECC at $18 \mathrm{~mm}$, as shown in Figure 1. The tangential stress coefficients of the internal and external surfaces of the ECC are shown in Figure 2. The shearing stress coefficient at the polar angle $\pi / 2$ is shown in Figure 3. The radial stress coefficient at the position of the minimum wall thickness is shown in Figure 4. The tangential stress of the ECC at the position of the minimum wall thickness is shown in Figure 5.

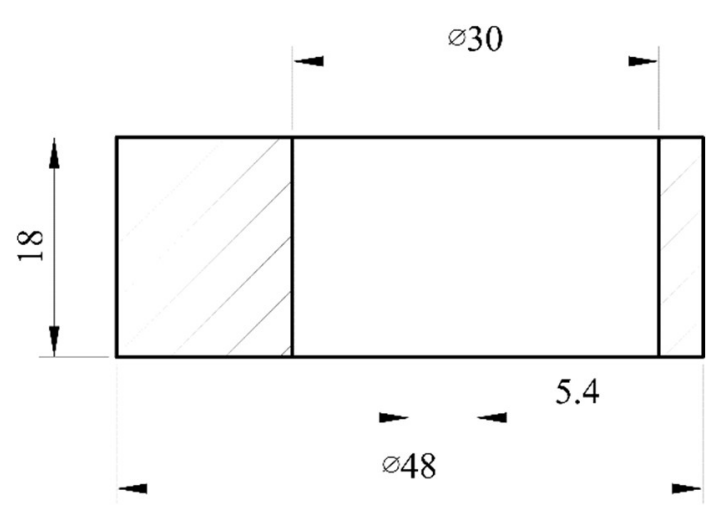

Figure 1. Part drawing of the eccentric cylindrical cam used as an example

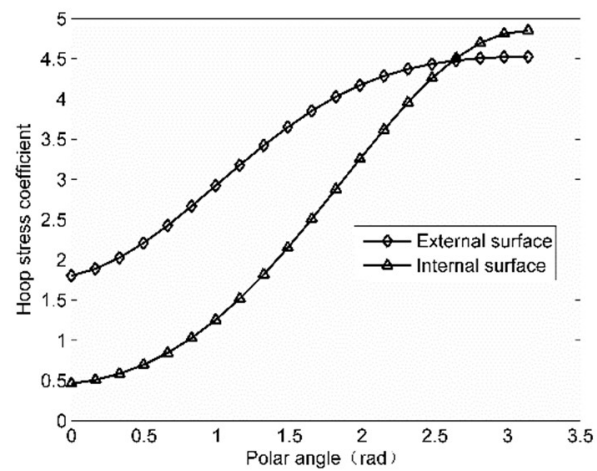

Figure 2. Tangential stress coefficients on the internal and external surfaces of the eccentric cylindrical cam

The tangential stress coefficient is always a positive value, indicating that the tangential stress of the cam is the tension stress owing to the expansion of the axial tube in the process of hydro-joining. The tangential stress coefficient on the external surface of the cam ranges from 0.46 to 4.85 and that on the inner surface ranges from 1.80 to 4.53 as the polar angle varies from 0 to $\pi$, which reaches a limiting value at the position of the minimum wall thickness. The tangential stress coefficient on the external surface of the cam is less than that on the inner surface when the polar angle is less than $152^{\circ}$. The radial stress coefficient at the position of the minimum wall thickness varies from 0 to -1 from the external surface to the internal surface of the cam owing to the expansion of the axial tube during hydro-joining. The variation of the tangential stress coefficient is nearly linear. The values of the tangential stress at the position of the minimum wall thickness on the external and internal surfaces of the cam are larger than radial the stress values by factors of 4.85 and 4.53, respectively The shearing stresses at the positions of maximum and minimum wall thicknesses of the cam under uniform inner pressure are equal to zero, and the curve is in the form of a parabola along the minimum wall thickness from the inner surface to the outer surface, which reaches a limiting value at the middle plane of the cam. The shearing stress value is only 0.0776 , which is $1.5 \%$ of the tangential stress value. The tangential stresses are respectively 242.6 and $226.3 \mathrm{MPa}$ at the internal and external surfaces at the position of the minimum wall thickness of the ECC. 




Figure 3. Shearing stress coefficient of the eccentric cylindrical cam at the polar angle $\pi / 2$

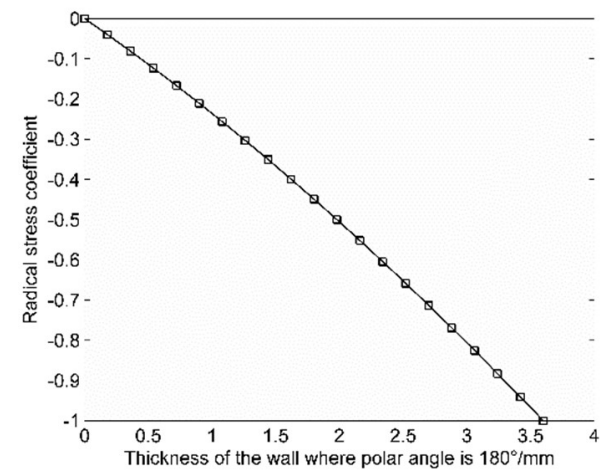

Figure 4. Radial stress coefficient of the ECC at the position of the minimum wall thickness

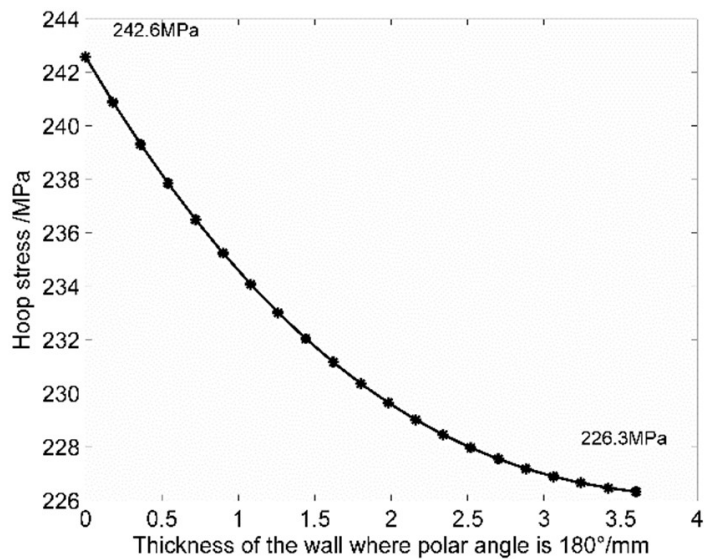

Figure 5. Tangential stress of the eccentric cylindrical cam at the position of the minimum wall thickness

\section{Numerical solutions of stresses of the ECC}

\subsection{Numerical analysis model}

The numerical analysis model of the stresses and deformations of the cam employed in the present study adopts the two-dimensional (2-D) PLANE82 8-node element, which is a higher order version of the 2-D, fournode element. The radial and tangential stresses of the
ECC under the action of a $20 \mathrm{MPa}$ hydrostatic pressure are shown in Figure 6.

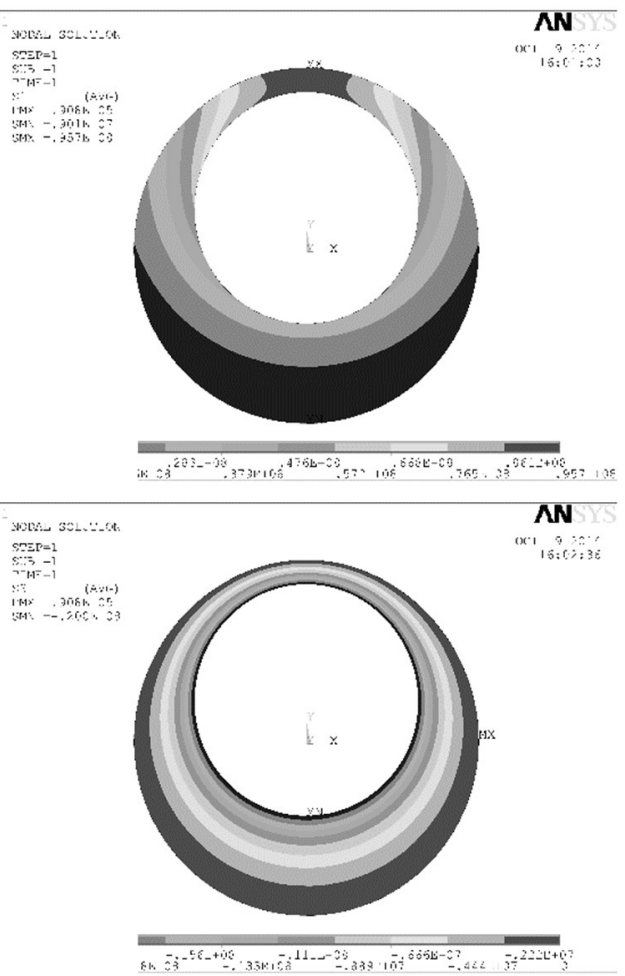
(a) Tangential stress
(b) Radial stress

Figure 6. Stresses of the eccentric cylindrical cam under a 20 MPa hydrostatic pressure applied to the internal surface

\subsection{Comparisons of the theoretical and numerical solutions of the stresses on the ECC}

Theoretical and numerical solutions of the tangential stresses on the internal and external surfaces of the ECC are shown in Figure 12 under the action of various internal pressures during hydro-joining of the cam and axial tube. The tangential stress of the cam is observed to linearly increase with increasing hydrostatic pressure. The theoretical and numerical solutions of the tangential stresses are $243 \mathrm{MPa}$ and $239 \mathrm{MPa}$, respectively, on the external surface at the position of the minimum wall thickness of the cam, and the error is only $1.6 \%$. The theoretical and numerical solutions of the tangential stresses are $226 \mathrm{MPa}$ and $224 \mathrm{MPa}$, respectively, on the internal surface, and the error is only $0.9 \%$. For an equivalent uniform internal pressure, the tangential stress on the external surface of is larger than that on the internal surface. The very small errors observed between the theoretical and numerical solutions are the result of the different sizes of meshing employed for the FEM, which can be decreased by meshing a smaller grid in the FEM.

\section{Conclusions}

The tangential stress and deformation of the ECC are a linear function of the uniform loads acting on the internal surface of the cam during hydro-joining. The radial and shearing stress coefficients determined for an example cam were far less than that of the tangential stress during 
hydro-joining. The tangential stress coefficient on the external surface was found to be greater than that on the inner surface for a polar angle greater than $152^{\circ}$. The maximum value of the tangential deformations on the internal and external surfaces of the cam are 2.49 and 8.27 $\mu \mathrm{m}$, respectively, under the action of an internal pressure of $50 \mathrm{MPa}$, which are located at the polar angles $42^{\circ}$ and $62^{\circ}$. Deformations in the radial and y direction at the position of the minimum wall thickness are 23.15 and $21.46 \mu \mathrm{m}$, respectively. Deformations in the radial and $\mathrm{x}$ direction are 16.59 and $12.15 \mu \mathrm{m}$, respectively, which are located in the polar angles $114^{\circ}$ and $96^{\circ}$, respectively. Under the action of a hydrostatic pressure of $50 \mathrm{MPa}$, the differences between the theoretical and numerical solutions of the tangential stress is $1.6 \%$ on the external surface of cam, and that of the deformation is $2.0 \%$ in the $y$ direction. The errors between the theoretical and numerical solutions of the stresses and deformations of the cam were found to be very small. Therefore, we conclude that the derived theoretical formulas can be applied to the failure analysis of an ECC during hydrojoining.

\section{References}

1. Liu Q, Ge JG and Liu G., et al. Analysis of stress and strain of hydro jointing for the PM and tube. Material Science and Technology 2007; 15: 614-618.

2. Lin YH, Qi X and Deng KH, et al. Emperical formula given for collapse resistance of worn casing under nonuniform loading. Oil Gas J 2011; 4: 60-64.

3. Zhou ZF, Wang XY and Gu JF. Numerical Simulation of Eccentric Cylinder Quenching Process. J Mech Eng 2011; 47: 62-67.

4. Zhong RB, Zhou J and Liu WH, et al. Theoretical investigation of a terahertz transmission line in bipolar coordinate system. Sci China 2012; 55: 35-42.

5. Zenkour AM. Stresses in rotating heterogeneous viscoelastic composite cylinders with variable thickness. App Math Mech 2011; 32: 507-520.

6. Liu MT, Li G, Li YQ, et al. Contact analysis between cam and rollers of inner-displaced indexing mechanism based on FEM[J]. Journal of Machine Design, 2015, 32(4): 14-18.

7. Wang XZ. Analysis on hydro-joining stress and deformation of eccentric cylindrical cam and tube[J]. Journal of Machine Design, 2019, 36(02): 88-95.

8. Dong MW, Shu X, Dai MH. Research on Modification Method of Convex Profile of Cylindrical Cam Continuous Transmission Mechanism [J]. Hoisting and Conveying Machinery, 2017(03): 26-29. 